Article

\title{
New strategies for efficient expression of heterologous sugar transporters in Saccharomyces cerevisiae
}

\author{
Marilia M. Knychala 1,2,3, Angela A. dos Santos ${ }^{1}$, Leonardo G. Kretzer ${ }^{1}$, Fernanda Gelsleichter ${ }^{1}$, Maria José \\ Leandro 2,3, César Fonseca 2,4, and Boris U. Stambuk 1,* \\ 1 Center of Biological Sciences, Department of Biochemistry, Federal University of Santa Catarina, \\ Florianópolis 88040-900, SC, Brazil; marilia.mknychala@gmail.com (M.M.K.); angela.asds@gmail.com \\ (A.A.d.S.); leo_kretzer@hotmail.com (L.G.K.); gelsnanda@gmail.com (F.G.) \\ 2 Laboratório Nacional de Energia e Geologia, I.P., Unidade de Bioenergia, Estrada do Paço do Lumiar 22, \\ 1649-038 Lisboa, Portugal; cesfonseca76@gmail.com (C.F.) \\ 3 ITQB NOVA, Instituto de Tecnologia Química e Biológica António Xavier, Av. da República, 2780-157 Oei- \\ ras, Portugal; mjose.leandro@itqb.unl.pt (M.J.L.) \\ 4 System Biology, Discovery, R\&D, Chr. Hansen A/S, Hørsholm, Denmark; \\ * Correspondence: boris.stambuk@ufsc.br; Tel.: +55-48-3721-4449
}

\begin{abstract}
In our previous work we had developed an hxt-null Saccharomyces cerevisiae strain displaying high xylose reductase, xylitol dehydrogenase and xylulokinase activities that proved to be useful as a chassis strain to study new xylose transporters, as SsXUT1 from Scheffersomyces stipitis. Spathaspora passalidarum and Spathaspora arborariae have in their genomes genes with high sequence similarity $(78-80 \%)$ to SsXUT1. To characterize these putative transporter genes (SpXUT1 and SaXUT1, respectively) they were expressed in the same chassis strain as SsXUT1. Surprisingly, the cloned genes could not restore the ability to grow in several monosaccharides tested, although the strains expressing the SsXUT1 and SpXUT1 permeases, after growth on maltose, showed the presence of ${ }^{14} \mathrm{C}$-glucose and ${ }^{14} \mathrm{C}$-xylose transport activity. An important feature of these permeases is that SsXUT1 lacks lysine residues in its N-terminal domain with high-confidence ubiquitinylation potential, and has only one at the C-terminal domain, while the SpXUT1 transporter had several of such residues at its C-terminal domain. When the SpXUT1 gene was cloned in a truncated version lacking such lysine residues, the permease allowed grow on glucose or xylose, and even promoted xylose fermentation by the $h x t$-null strain. In another approach, we deleted two arrestins known to be involved in sugar transporter ubiquitinylation and endocytosis (ROD1 and ROG3), but only the rog3 $\Delta$ strain allowed modest growth on these sugars. Taken together, these results suggest that to allow efficient sugar transporter expression in S. cerevisiae the lysines involved in transporter endocytosis should be removed from the sequence of the permease.
\end{abstract}

Keywords: xylose; hxt-null; ubiquitinylation; lysine; truncated permease; endocytosis; XUT1; ROD1, ROG3

\section{Introduction}

The development and use of sustainable alternatives to fossil fuels has been ongoing for the last few decades, and the use of ethanol as a renewable fuel has intensify specially in the USA and Brazil, which account for over $80 \%$ of the global ethanol production [1-4]. Presently, three generations of biofuels are being implemented: first generation (1G) biofuels from food based plant materials; second generation $(2 \mathrm{G})$ biofuels from lignocellulosic biomasses; and third generation (3G) biofuels from algae-based biomasses [5]. Bioethanol is produced by fermentation of the sugars present in the above biomasses, particularly those present in lignocellulosic plant materials obtained from agricultural and forestry residues, which exists in large quantities and prevails on Earth, and does not contribute to the food vs. fuel controversy $[4,6]$. These sugars can be used to produce not 
only ethanol, but also other valuable chemical products. Biochemical conversion of lignocellulosic biomass into ethanol involves several steps including pre-treatment, enzymatic hydrolysis and fermentation, being this last step a major bottleneck to economically feasible $2 \mathrm{G}$ ethanol production $[7,8]$.

Lignocellulosic biomass is composed of cellulose (a linear polymer of glucose molecules linked by $\beta 1-4$ glycosidic bounds), hemicellulose (a branched and highly heterogeneous polymer containing both hexoses and pentoses), and lignin. Thus, the resulting biomass hydrolysates have various hexoses and pentoses available, and after glucose, xylose and arabinose are the second and third most abundant sugars in plant biomass hydrolysates $[9,10]$. While glucose can be easily fermented by industrial Saccharomyces cerevisiae strains [11], this yeast is not naturally able to ferment the pentoses xylose or arabinose, unless it is genetically modified to express the assimilation routes for these sugars [12-14]. In the case of xylose, genes encoding for xylose reductase (XR) and xylitol dehydrogenase (XDH) from Scheffersomyces stipitis or Spathaspora passalidarum, or xylose isomerase (XI) from bacteria and fungi have been extensively used [14-18]. Since both pathways transform xylose into xylulose, it is also required to overexpress the endogenous xylulokinase $(\mathrm{XK})$ gene that will enhance the entrance of this sugar into the pentose-phosphate pathway. While the XI pathway can provide higher ethanol yields, the $\mathrm{XR} / \mathrm{XDH}$ route allows higher ethanol productivities and higher xylose consumption rates by engineered $S$. cerevisiae yeasts $[19,20]$.

When the metabolic pathway for xylose is established intracellularly, the transport of the sugar across the plasma membrane becomes the limiting step in the assimilation and fermentation of this pentose. The uptake of xylose in S. cerevisiae is mediated by a large family of hexose transporters, encoded by the HXT1-HXT17 and GAL2 genes, that have significant lower affinities for this pentose than for their main substrate glucose $[12,21]$. Thus, the discovery and heterologous expression of pentose transporters in $S$. cerevisiae has also being one of the alternatives used to improve $2 \mathrm{G}$ ethanol production [22-27]. Consequently, the engineering of pentose transporters in recombinant S. cerevisiae yeasts is the subject of several recent reviews in the scientific literature [28-31]. A suitable platform to clone and characterize monosaccharide transporters was created in 1999 by deleting all known hexose transporters in S. cerevisiae, as this $h x t^{0}$-null EBY.VW4000 strain is not able to grow on media with glucose, fructose or mannose as the sole carbon source (although it still grows very slowly on galactose), but grows normally on maltose as this sugar is transported by different maltose-specific permeases encoded by MALx1 genes [32,33]. This $h x t^{0}$-null strain has being used to characterize hundreds of hexose transporters from other yeasts, fungi, plants, and other eukaryotes including human GLUT transporters, as well as xylose transporters, after the introduction of a xylose utilization pathway in this $h x t^{0}$-null strain [24,34]. One drawback of this $h x t^{0}$-null EBY.VW4000 strain is that it required 16 successive deletion rounds with the LoxP/Cre system, which resulted in gene losses and several chromosomal rearrangements, affecting, for example, sporulation and spore germination by this $h x t^{0}$-null strain [35]. Nevertheless, a recent publication shows that using CRISPR-SpCas9 technology is possible to construct an $h x t^{0}$-null strain without these drawbacks [36].

Some years ago our group used an $h x t$-null strain, deleted in the principal hexose transporters ( $h x t 1 \Delta$ to $h x t 7 \Delta$ and gal2 $\Delta$ ) and thus unable to grow on glucose, fructose and other monosaccharides, as a platform to study xylose transport because it had high xylose reductase, xylitol dehydrogenase and xylulokinase activities. This DLG-K1 strain was used to characterize the xylose fermentation capacity of yeast strains expressing individual hexose transporters (e.g. HXT1, HXT2, HXT5 and HXT7) from S. cerevisiae [21], and allowed the cloning and characterization of three novel xylose transporters (XUT1, QUP2 and HXT2.6) from Scheffersomyces stipitis [27]. Results showed that the SsXUT1 gene allowed ethanol production from xylose or xylose plus glucose as carbon sources, while the SsHXT2.6 permease produced both ethanol and xylitol, and the strain expressing the SsQUP2 gene produced mainly xylitol during xylose consumption. Since the 
genome of other new xylose-fermenting yeast species (e.g. Spathaspora passalidarum $[37,38]$ or $S p$. arborariae $[39,40])$ have genes encoding transporters with high sequence similarity $(78-80 \%)$ to SsXUT1, we decided to clone and characterize these putative permeases with our hxt-null DLG-K1 strain. In the present work we show that the cloned genes could not restore the ability of the yeast strain to grow in several monosaccharides tested, although the strains expressing the SsXUT1 and SpXUT1 permeases, after growth on maltose, showed ${ }^{14} \mathrm{C}$-glucose and ${ }^{14} \mathrm{C}$-xylose transport activity. Thus, we analyzed the presence of lysine residues in the $\mathrm{N}$ - or C-terminal domain of these transporters with ubiquitinylation potential, as well as the involvement of two $\alpha$-arrestins known to be involved in sugar transporter ubiquitinylation and endocytosis in S. cerevisiae. Our results indicate that to allow efficient sugar transporter expression in S. cerevisiae the lysines involved in transporter endocytosis should be ideally removed from the sequence of the permease.

\section{Materials and Methods}

\subsection{Strains, media and growth conditions}

The yeast strains and plasmids used in this study are listed in Table 1. The Escherichia coli strain $\mathrm{DH} 5 \alpha$ was used for cloning, and was grown in Luria broth (1\% tryptone, $0.5 \%$ yeast extract, $0.5 \%$ sodium chloride) supplemented with $100 \mathrm{mg} / \mathrm{L}$ ampicillin. Yeasts were grown on rich YP medium (1\% yeast extract, $2 \%$ Bacto peptone, Sigma-Aldrich Brasil Ltda., São Paulo, SP, Brazil), synthetic complete (YNB) medium $(0.67 \%$ yeast nitrogen base without amino acids, supplemented with adequate auxotrophic requirements, Sigma-Aldrich), or optimized [41] YNB medium (o-YNB, containing 1.34\% yeast nitrogen base without amino acids, supplemented with $2 x$ adequate auxotrophic requirements and $1.5 \%$ casaminoacids, Sigma-Aldrich), with $2 \%$ maltose, glucose or xylose as carbon source. When required, 2\% Bacto agar, $0.5 \mathrm{~g} / \mathrm{L}$ zeocin (Invivogen, San Diego, CA, USA) or $200 \mathrm{mg} / \mathrm{L}$ geneticin sulfate (G-418, Sigma-Aldrich) were added to the medium. The $\mathrm{pH}$ of the medium was adjusted to $\mathrm{pH} 5.0$ with $\mathrm{HCl}$, or to $\mathrm{pH} 8.0$ (adjusted with $\mathrm{NaOH}$ ) when zeocin was used. Cells were pre-grown in YNB-2\% maltose and used to inoculate new medium containing the carbon sources tested with an initial cell density of 0.1 A600nm (Cary 60 UV-VIS spectrophotometer, Agilent Technologies, Santa Clara, CA, USA). Growth was performed aerobically in cotton plugged Erlenmeyer flasks filled to $1 / 5$ of the volume with medium at $28^{\circ} \mathrm{C}$ with $160 \mathrm{rpm}$ orbital shaking. Cellular growth was followed by absorbance measurements at $600 \mathrm{~nm}(A 600 \mathrm{~nm})$, and culture samples were harvested regularly, centrifuged $\left(5,000 \mathrm{~g}, 1 \mathrm{~min}\right.$ at $\left.4^{\circ} \mathrm{C}\right)$, and supernatants used for the quantification of substrates and fermentation products as described below. Alternatively, yeast strains were grown in $100 \mu \mathrm{l}$ of o-YNB medium (lacking uracil) containing $2 \%$ maltose, glucose or xylose at $30^{\circ} \mathrm{C}$ in 96 -well plates in a Tecan INFINITY M200 PRO microplate reader (Tecan Austria GMBH, Grodig, Austria). All wells in the plate were tightly sealed with AccuClear Sealing Film for qPCR (E \& K Scientific, Santa Clara, CA, USA), and growth of each culture was monitored by measuring the $A 600 \mathrm{~nm}$ every $10 \mathrm{~h}$, with high intensity orbital shaking between measurements. After $\sim 190 \mathrm{~h}$ of growth the plates were centrifuged $\left(3,500 \mathrm{~g}, 10 \mathrm{~min}\right.$ at $\left.4^{\circ} \mathrm{C}\right)$, and the supernatants used for ethanol determination as described below. For batch fermentations, cells were pre-grown in synthetic complete YNB medium containing $2 \%$ maltose for 20 hours at $28^{\circ} \mathrm{C}$, the cells were collected by centrifugation at $6,000 \mathrm{~g}$ for $5 \mathrm{~min}$ at $4^{\circ} \mathrm{C}$ and washed twice with sterile water, and inoculated at a high cell density $(10.0 \pm 0.5 \mathrm{~g}$ dry cell weight (DCW)/L) into 25 $\mathrm{mL}$ of synthetic YNB medium containing $2 \%$ glucose or xylose. Batch fermentations were performed at $30^{\circ} \mathrm{C}$ in closed $50-\mathrm{ml}$ bottles with a magnetic stir bar to allow mild agitation $(100 \mathrm{rpm})$. Samples were collected regularly and processed as described above.

Table 1. Yeast strains, plasmids and primers used in this study.

Strains, plasmids and primers Relevant features, genotype or sequence Source 


\begin{tabular}{|c|c|c|}
\hline Yeast strains: & & \\
\hline Sp. arborariae UFMG-HM19.1 $\mathrm{A}^{\mathrm{T}}$ & Isolated from rotting wood in Minas Gerais, Brazil & [39] \\
\hline Sp. passalidarum UFMG-CM-Y474 & Isolated from rotting wood in Roraima, Brazil & [17] \\
\hline \multirow[t]{2}{*}{ S. cerevisiae DLG-K1 } & 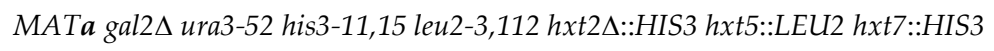 & [21] \\
\hline & 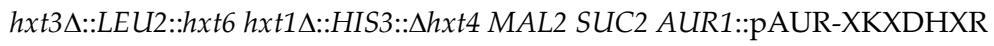 & \\
\hline S. cerevisiae DLG-K1 $\triangle \mathrm{R} 1$ & Isogenic to DLG-K1, but rod1 $\Delta:: L o x P-K a n M X 6-L o x P$ & This work \\
\hline S. cerevisiae $\mathrm{DLG}-\mathrm{K} 1 \Delta \mathrm{R} 3$ & Isogenic to DLG-K1, but rog3 $\Delta::$ LoxP-Ble $e^{\mathrm{R}}$ LoxP & “ \\
\hline S. cerevisiae DLG-K1 $\Delta \mathrm{R} 1 \Delta \mathrm{R} 3$ & Isogenic to DLG-K1, but rog3 $\Delta:$ :LoxP-Ble $e^{\mathrm{R}}$ LoxP $\operatorname{rod} 1 \Delta::$ LoxP-KanMX6-LoxP & “ \\
\hline \multicolumn{3}{|l|}{ Plasmids: } \\
\hline pUG6 & LoxP-PтеF-KanMX6-Ttef-LoxP & [42] \\
\hline pUG66 & LoxP-PтеF-Ble $e^{R}$-Ttef-LoxP & $"$ \\
\hline pPGK & $2 \mu U R A 3$ PPGK1-TPGK1 & {$[43]$} \\
\hline pGPD-426 & 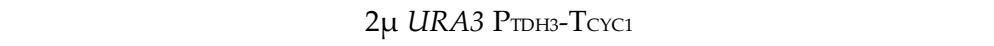 & {$[44]$} \\
\hline pPGK-SsXUT1 & $2 \mu$ URA3 PPGK1-SsXUT1-ТPGK1 & [27] \\
\hline pPGK-SpXUT1 & $2 \mu U R A 3$ PPGK1-SpXUT1-ТPGK1 & This work \\
\hline pPGK-SpXUT1 $\Delta \mathrm{C}$ & $2 \mu U R A 3$ PPGK1-SpXUT1 $\Delta \mathrm{C}-$-TPGK1 & $"$ \\
\hline pGPD-SaXUT1 & $2 \mu$ URA3 РтDH3-SaXUT1-Тсус1 & $"$ \\
\hline pGPD-SaXUT1 $1 \Delta \mathrm{NC}$ & $2 \mu U R A 3$ PTDH3-SaXUT1 $_{\text {NNC-Tcrc1 }}$ & $"$ \\
\hline \multicolumn{3}{|l|}{ Primers: ${ }^{1}$} \\
\hline pPGK-SpXUT1-F & AGATCGGAATTCAAGCTTATGCACGGAGGTTCAGACG & “ \\
\hline pPGK-SpXUT1-R & GCCGGATCCGGCTTAAGCACTGTCAGCATCAGC & $"$ \\
\hline pPGK-SpXUT1 $1 \Delta \mathrm{C}-\mathrm{R}$ & GGCGGATCCAAATTAGTCAGAGTCTAATTCTTCTCCGCC & “ \\
\hline pGPD-SaXUT1-F & AGATCGGAATTCAAGCTTGGATCCATGCACGGAGGTTCAGATAGTAA & $"$ \\
\hline pGPD-SaXUT1-R & GCCCTCGAGGTCGACCCCGGGGGCTTAATCAGCATCAGCAACCTTTTC & $"$ \\
\hline pGPD-SaXUT1 $1 \Delta \mathrm{NC}-\mathrm{F}$ & GCCGGATCCAAAATGCGTTTAGAAATCGCCGGTAAACC & $"$ \\
\hline pGPD-SaXUT1 $1 \Delta$ NC-R & GCCCTCGAGGTCGACTTAATCAGAATCTAAGTCTTCTAATCC & $"$ \\
\hline $\mathrm{ROD} 1 \Delta-\mathrm{F}$ & ATGTTTTCATCATCATCTCGACCTTCAAAAGAGCCATTACCCAGCTGAAGCTTCGTACGC & $"$ \\
\hline $\mathrm{ROD} 1 \Delta-\mathrm{R}$ & CTATGAGCGATCCCGTTTTGTGAACATCTCCATTAAATTAGCATAGGCCACTAGTGGATC & $"$ \\
\hline $\mathrm{ROG} 3 \Delta-\mathrm{F}$ & GGCGTTGATAAAGAGCCAATATCTATTGTTGCTACATAGACCAGCTGAAGCTTCGTACGC & $"$ \\
\hline $\mathrm{ROG} 3 \Delta-\mathrm{R}$ & CGACTATCGTTTGTTACCCTTTGATAGAAAACCTCCCATAGCATAGGCCACTAGTGGATC & “ \\
\hline V-ROD1-F & AGTCGAGTCCCTTGGTACAT & “ \\
\hline V-ROD1-INT-F & CTGCCGTCACTTATGCTCTG & “ \\
\hline V-ROD1-R & CGAATGATGTCTGTGGGATC & “ \\
\hline V-ROG3-F & GCAAGTACAGAGTCCTACCA & “ \\
\hline V-ROG3-INT-F & CTGTGTGCAAGATTGTGATG & “ \\
\hline V-ROG3-R & GCCAGTTAGAGTGCGTAAAT & “ \\
\hline $\mathrm{V}-\mathrm{Kan}^{\mathrm{R}}-\mathrm{F}$ & CCGGTTGCATTCGATTCC & $"$ \\
\hline V-Ble ${ }^{R}-F$ & ССTTCTATGAAAGGTTGGGC & $"$ \\
\hline
\end{tabular}

${ }^{1}$ Bold sequences indicate restriction enzyme sites (BamHI, EcoRI or Xho1) used for cloning, underlined sequences allow amplification of genes or the transformation modules present in plasmids pUG6 and pUG66, and italicized sequences are homologous to the upstream and downstream region of the target genes that were deleted. 
Standard methods for DNA manipulation and analysis, as well as bacterial and yeast transformation, were employed [45,46]. The genomic DNA from the $S p$. passalidarum and Sp. arborariae strains was purified using a YeaStar Genomic DNA kit (Zymo Research, Irvine, CA, USA). Based on the genome sequence of Sp. arborariae and Sp. passalidarum [38,40], primers were designed (Table 1) to amplify genes with high sequence homology to the XUT1 gene from Sc. stipitis (SsXUT1 [27]), introducing restriction sites for cloning into multicopy shuttle vectors containing strong and constitutive promoters and terminators (pPGK and p423-GPD, Table 1) as well as the URA3 gene used as selective marker. Alternatively, the SpXUT1 gene was amplified using a reverse primer (pPGK-SpXUT1 $\triangle \mathrm{C}-\mathrm{R}$ ) that introduces a premature stop codon and removes the last 22 amino acid residues of the protein. In the case of the SaXUT1 gene the permease was truncated both in the N- and C-terminal domains, removing the first 17 amino acid residues (primer pGPD-SaXUT1 $\triangle \mathrm{NC}-\mathrm{F}$, which introduces a methionine after the 17 residues deleted), and removing the last 18 amino acid residues (primer pGPD-SaXUT1 $\triangle N C-R$ ) as described for the SpXUT1 permease.

In another approach, we deleted the ROD1 and/or ROG3 genes in strain DLG-K1 using the PCR-based gene replacement procedure [42]. Briefly, the LoxP-KanMX6-LoxP knockout cassette from plasmid pUG6 (Table 1) was amplified with primers ROD1 $\Delta-\mathrm{F}$ and ROD1 $\Delta-\mathrm{R}$ (Table 1), and the resulting PCR product of 1,615-bp (flanked by $\sim 40$ bp of homology to the upstream and downstream regions of the ROD1 gene) containing the KanMX6 gene was used to transform competent yeast cells. After 2-hour cultivation on YP-2\% glucose, the cells were plated on the same medium containing G-418 and incubated at $28^{\circ} \mathrm{C}$. G-418-resistant isolates were tested for proper genomic integration of the LoxP-KanMX6-LoxP cassette at the ROD1 locus by diagnostic colony PCR using 4 primers (V-ROD1-F, V-ROD1-INT-F, V-ROD1-R and V-Kan ${ }^{\mathrm{R}}-\mathrm{F}$; Table 1). This set of primers amplified a 3,556-bp fragment (primers V-ROD1-F and V-ROD1-R) or a 2,633-bp fragment (primers V-ROD1-INT-F and V-ROD1-R) from a normal ROD1 locus, or yielded a 2,720-bp fragment (primers V-ROD1-F and V-ROD1-R) and a 1,500-bp fragment (primers $\mathrm{V}-\mathrm{Kan}^{\mathrm{R}}-\mathrm{F}$ and V-ROD1-R) if the LoxP-KanMX6-LoxP cassette was correctly integrated at the ROD1 locus, producing strain DLG-K1 $1 \mathrm{R} 1$ (rod1 $\Delta::$ LoxP-KanMX6-LoxP, Table 1). A similar approach was used to delete the ROG3 gene from strains DLG-K1 or DLG-K1 $\triangle \mathrm{R} 1$.

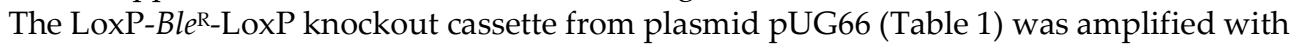
primers ROG $3 \Delta-\mathrm{F}$ and ROG3 $\Delta-\mathrm{R}$ (Table 1), and the resulting PCR product of 1,265-bp (flanked by $\sim 40 \mathrm{bp}$ of homology to the upstream and downstream regions of the ROG3 locus) containing the Ble gene was used to transform competent cells. After 2-hour cultivation on $\mathrm{YP}-2 \%$ glucose, the transformed cells were plated on the same medium containing zeocin and incubated at $28^{\circ} \mathrm{C}$. Zeocin-resistant isolates were tested for proper genomic integration of the LoxP-Ble $e^{\mathrm{R}}-\mathrm{LoxP}$ cassette at the ROG3 locus by diagnostic colony PCR using 4 primers (V-ROG3-F, V-ROG3-INT-F, V-ROG3-R and V-BleR-F; Table 1). This set of primers amplified a 2,967-bp fragment (primers V-ROG3-F and V-ROG3-R) or a 1,249-bp fragment (primers V-ROG3-INT-F and V-ROG3-R) from a normal ROG3 locus, or yielded a 1,757-bp fragment (primers V-ROG3-F and V-ROG3-R) and a 750-bp fragment (primers V-Ble ${ }^{\mathrm{R}}-\mathrm{F}$ and $\mathrm{V}-\mathrm{PHO} 13-\mathrm{R}$ ) if the LoxP-Ble $\mathrm{R}-L o x P$ module replaced and de-

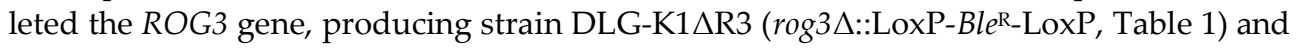
strain DLG-K1 $\Delta \mathrm{R} 1 \Delta \mathrm{R} 3$ (rod1 $1:$ :LoxP-KanMX6-LoxP and $\operatorname{rog} 3 \Delta::$ LoxP-Ble ${ }^{\mathrm{R}}-\mathrm{LoxP}$, Table 1).

\subsection{Transport assays}

The DLG-K1 yeast strain transformed with plasmids pPGK-SsXUT1 or pPGK-SpXUT1 were grown in YNB-2\% maltose to mid-log phase (A600nm of 0.6-1.0), centrifuged, washed twice with cold distilled water, and suspended in water to a cell density of $20 \mathrm{~g} \mathrm{DCW} / \mathrm{L}$. The uptake of D-[U-14C]glucose or D-[U-14C]xylose (both from Amersham, Little Chalfont, UK) was determined by placing $20 \mu \mathrm{L}$ of the yeast suspension with $20 \mu \mathrm{L}$ of $100 \mathrm{mM}$ Tris-citrate buffer, $\mathrm{pH}$ 5.0, in the bottom of 8-mL Röhren tubes (Sarstedt AG \& Co. KG, Numbrecht, Germany). The tube was incubated at $25{ }^{\circ} \mathrm{C}$ for $5 \mathrm{~min}$, and the 
reaction started by adding $10 \mu \mathrm{L}$ of the radiolabelled substrate $(102-104 \mathrm{cpm} / \mathrm{nmol})$ at the desired final sugar concentration with vigorous shaking. After $5 \mathrm{~s}$ the reaction was stopped with $5 \mathrm{~mL}$ of ice-cold distilled water and vigorous shaking, immediately filtered in Whatman glass microfiber GF/C membranes (2.4 cm diameter), and the filters washed twice with $10 \mathrm{~mL}$ of ice-cold distilled water. The filters were placed into scintillation vials containing $6 \mathrm{ml}$ of liquid scintillation cocktail (OptiPhase 'HiSafe' 2, Wallac, Turku, Finland), and the radioactivity retained on filters was counted using a liquid scintillation counter (Tri-Carb ${ }^{\text {TM }} 1600$ CA, Packard, Downers Grove, IL, USA). The kinetic parameters ( $K_{\mathrm{m}}$ and $V_{\mathrm{max}}$ ) of glucose and xylose transport by the cloned permeases were determined by fitting the experimental data to the Michaelis-Menten equation, using SigmaPlot v. 11.0 (Systat Software Inc., San Jose, CA, USA).

\subsection{Analytical Methods}

Glucose, xylose, ethanol, xylitol, and glycerol were determined by high performance liquid chromatography (Prominence HPLC system) equipped with a RID-20A refractive index detector (Shimadzu Co., Tokyo, Japan) using an Aminex HPX-87H column (Bio-Rad Laboratories, Hercules, CA, USA). The HPLC apparatus was operated at $50^{\circ} \mathrm{C}$ using $5 \mathrm{mM} \mathrm{H}_{2} \mathrm{SO}_{4}$ as mobile phase at a flow rate of $0.6 \mathrm{~mL} / \mathrm{min}$, and $10 \mu \mathrm{L}$ injection volume.

\section{Results}

The SsXUT1 permease from Ss. stipitis has been studied previously in S. cerevisiae, showing the highest preference for xylose over glucose among several other transporters analyzed [24,27]. Using the sequence of this transporter with 566 amino acids allowed the identification of two other putative permeases with high sequence identity from the $x y-$ lose-fermenting yeasts $S p$. passalidarum and $S p$. arborariae. The open-reading frame (ORF) from the $S p$. passalidarum genome encodes for a transporter with 576 amino acids and $78.8 \%$ sequence identity with SsXUT1, while the ORF present in the genome of Sp. arborariae encodes for a transporter with 572 amino acids and $79.51 \%$ sequence identity with SsXUT1. These genes were cloned into multicopy plasmids as described in the Materials and Methods section, and named SpXUT1 and SaXUT1, respectively. To characterize these putative transporters the DLG-K1 ( $h x t$-null) strain transformed with plasmids containing these genes were pre-grown on YNB-2\% maltose, and inoculated (initial A600nm of 0.1 ) into YNB medium containing $2 \%$ glucose or xylose. However, no significant growth or sugar consumption was observed during $120 \mathrm{~h}$ of incubation for both permeases when expressed in strain DLG-K1. Figure 1 shows as example the data for strain DLG-K1 transformed with plasmid pPGK-SpXUT1. Absence of growth was also observed when the cells were inoculated in media containing $2 \%$ fructose or galactose (data not shown).

These were quite surprising results since the SpXUT1 permease expressed in strain DLG-K1, after growth on $2 \%$ maltose, showed a clear ${ }^{14} \mathrm{C}$-glucose and ${ }^{14} \mathrm{C}$-xylose transport activity similar to the transport activity present in the same $h x t$-null strain expressing the SsXUT1 permease (Figure 2). The SsXUT1 permease allows ${ }^{14} \mathrm{C}$-glucose transport with a $K_{\mathrm{m}}$ of $24.5 \mathrm{mM}$, but with a low $V_{\max }$, while ${ }^{14} \mathrm{C}$-xylose transport occurs with a much lower affinity, although with higher capacity (Figure 2, Table 2). In the case of the $S p X U T 1$ permease ${ }^{14} \mathrm{C}$-glucose transport is mediated with a similar affinity of 26.1 $\mathrm{mM}$, and also a low $V_{\max }$, and ${ }^{14} \mathrm{C}$-xylose transport is also mediated with an even lower affinity but high capacity, when compared to glucose uptake by this permease expressed in strain DLG-K1 (see Table 2). 

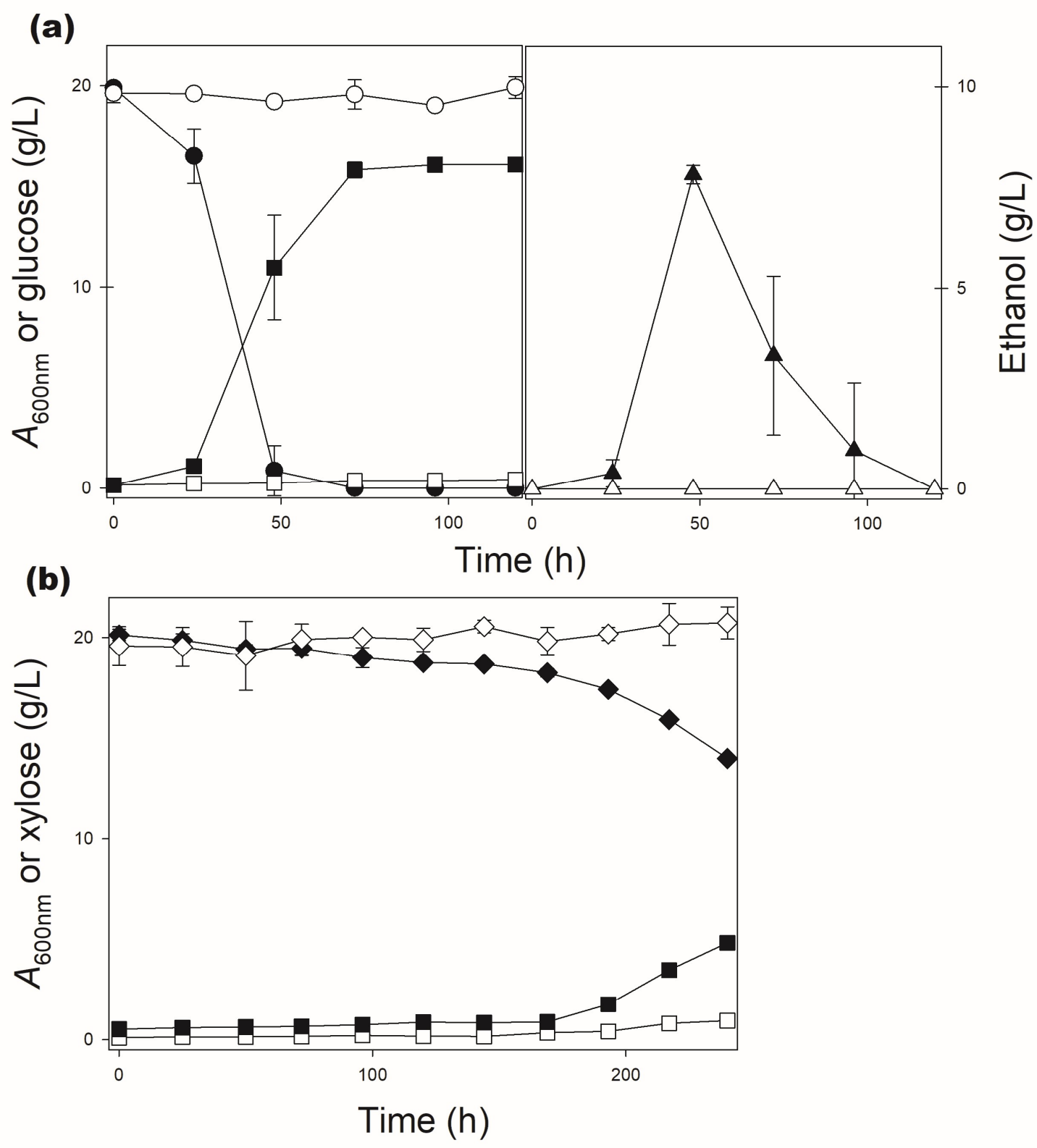

Figure 1. Growth of strain DLG-K1 transformed with plasmid pPGK-SpXUT1 (open symbols) or plasmid pPGK-SpXUT1 $\triangle \mathrm{C}$ (black symbols) on YNB medium (lacking uracil) containing $2 \%$ glucose (a) or xylose (b) as carbon source. At the indicated time points the cell growth (squares), glucose (circles), xylose (diamonds) and ethanol (triangles) concentrations were determined. No ethanol was produced during xylose consumption.

Table 2. Kinetic parameters of ${ }^{14} \mathrm{C}$-glucose and ${ }^{14} \mathrm{C}$-xylose transport by the SsXUT1 and SpXUT1 permeases.

\begin{tabular}{|c|c|c|c|c|}
\hline \multirow[b]{3}{*}{ Strain: } & \multicolumn{2}{|c|}{ Transport of ${ }^{14} \mathrm{C}$-Glucose } & \multicolumn{2}{|c|}{ Transport of ${ }^{14} \mathrm{C}-\mathrm{Xylose}$} \\
\hline & & $V_{\max }$ & & $V_{\max }$ \\
\hline & $K_{\mathrm{m}}(\mathrm{mM})$ & $\left(\mathrm{mmol} \mathrm{h}^{-1} \mathrm{gDCW}^{-1}\right)$ & $K_{\mathrm{m}}(\mathrm{mM})$ & $\left(\mathrm{mmol} \mathrm{h}^{-1} \mathrm{gDCW}^{-1}\right)$ \\
\hline DLG-K1 + pPGK-SsXUT1 & $24.5 \pm 4.1$ & $10.8 \pm 1.8$ & $417.7 \pm 176$ & $72.4 \pm 27$ \\
\hline DLG-K1 + pPGK-SpXUT1 & $26.1 \pm 11.5$ & $4.3 \pm 1.9$ & $711 \pm 550$ & $72.3 \pm 7.5$ \\
\hline
\end{tabular}




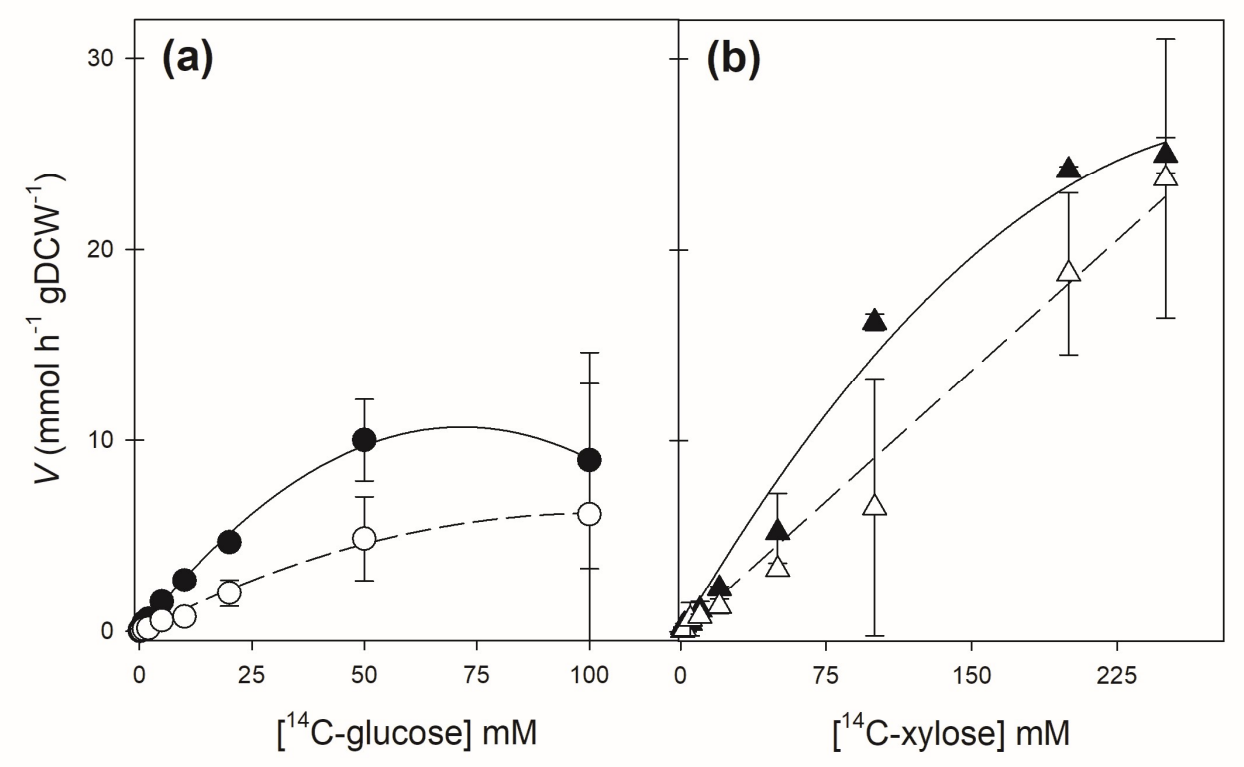

Figure 2. Kinetics of glucose (a) or xylose (b) transport by strain DLG-K1 transformed with plasmid pPGK-SsXUT1 (black symbols) or plasmid pPGK-SpXUT1 (open symbols). Cells pre-grown in $2 \%$ maltose were used to determine the initial rates of uptake of the indicated labeled sugar concentrations as described in Materials and Methods.

Despite the high sequence identity between these three transporters, a significant difference was observed in their N- and C-terminal cytoplasmic domains regarding the presence of lysine residues with high-confidence ubiquitinylation potential (Figure 3). In the case of the SsXUT1 permease it does not have any lysine in its $\mathrm{N}$-terminal domain, and has a single one with high-confidence ubiquitinylation potential at the end of the C-terminal domain (residue K-558). The permease that had the higher number of lysine residues at their cytoplasmic domains was the SpXUT1 transporter, since this permease had a medium-confidence possible ubiquitinylated lysine at its $\mathrm{N}$-terminal domain (residue K-17), and also had 3 lysine residues at its C-terminal cytoplasmic domain with high-confidence ubiquitinylation potential (residues K-555, K-557 and K-567, see Figure 3). In the case of the SaXUT1 permease the lysine residues with ubiquitinylation potential were the same as the SpXUT1 permease, although residues K-555 and K-567 were considered with medium-confidence ubiquitinylation potential by the UbPred program used to identify such residues [48].

A

SSXUT1 SPXUT1 SaXUT1

B

SSXUT1 SPXUT1 SaXUT1
$\mathrm{TM}-\mathrm{I}$

MHGGGDGNDITEIIAARRLQIAGKSGVAGLVANSRSFFIAVFA.SLGGLVYGYNQGMFGQISGMYSFSKAIGVEKIQDNP MHGGSDGNDVQAIIAOKRLEIAGKPGIAGLIANRKSFLIAVFASLGGLVYGYNQGMFGQISGMTSFSA.A.AGVGKIQDNP MHGGSDSNDIQQI IAEKRLEIAGKPGIAGLVANKKSFLIAVFASLGGLVYGYNQGMFGQISGMTSFSEAAGVGKIQDNP

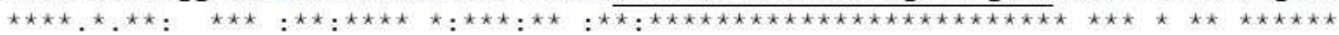

\section{TM-XII}

AYVQFFCPETKGRTLEEIDELFGDTSGTSKMEKEIHEQKLKEVGLLQLIGEENASESENSKADVYHVEK AYVFFFCPETKGRTLDE I DELFGDTSGVSKREGEIRNRILKEVGLLELIGLEELDSDKSKGGDVHYQEEKAADADADSA AYVLFFVPETKGRTLDEIDELFGDTSGVSKREAEIHNRI IKEVGLLELVGLEDLDSDKSKGGDVQYQEEKVADAD

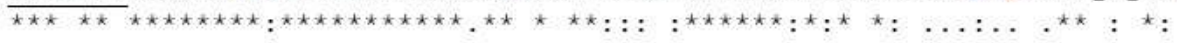

Figure 3. Sequence alignment of the N-terminal (A) and C-terminal (B) cytoplasmic domains of the SsXUT1, SpXUT1 and SaXUT1 transporters. The protein sequences were aligned using Clustal Omega [47] and the lysine residues with medium(blue) or high-confidence (red) ubiquitinylation potential were determined with the UbPred program [48]. 
Considering that such lysine residues with ubiquitinylation potential could be involved in removing the transporters from the plasma membrane through endocytosis [49], we decided to remove these terminal lysine residues by simply truncating the permeases. In the case of the SpXUT1 permease we removed the last 22 amino acid residues of the protein by introducing a premature stop codon during cloning as described in

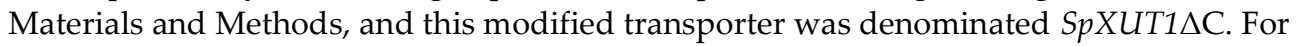
the SaXUT1 permease we not only removed the last 18 amino acid residues, but also the first 17 amino acid residues, producing the $\operatorname{SaXUT1\triangle N} \Delta \mathrm{C}$ permease that lacks all lysine residues with ubiquitinylation potential from their $\mathrm{N}$ - and C-terminal domains.

As can be seen in Figure 1, when the DLG-K1 strain was transformed with the pPGK-SpXUT1 $\triangle \mathrm{C}$ plasmid the cells were able to consume, growth on and ferment efficiently glucose (Figure 1a), and consumption of xylose also occurred, although was delayed and incomplete (Figure 1b). Nevertheless, this strain expressing the SpXUT1 $\triangle \mathrm{C}$ permease was able to ferment xylose efficiently, producing both ethanol and xylitol, while the strain expressing the full-length transporter (SpXUT1) was unable to consume the pentose under fermentative conditions (Figure 4). In the case of the SaXUT1 permease truncated in both the $\mathrm{N}$ - and C-terminal domains $(\operatorname{SaXUT1} \Delta \mathrm{N} \Delta \mathrm{C})$ we also observed clear improvements in growth on glucose or xylose, but not as impressive as with

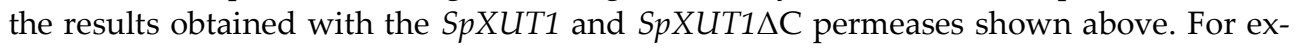
ample, under the fermentative conditions shown in Figure 4, after $48 \mathrm{~h}$ the DLG-K1 strain transformed with plasmid pGPD-SaXUT1 $\triangle \mathrm{N} \Delta \mathrm{C}$ consumed only $\sim 5 \mathrm{~g} / \mathrm{L}$ of xylose, producing $\sim 1.5 \mathrm{~g} / \mathrm{L}$ of ethanol, while the strain expressing the full length SaXUT1 permease was unable to consume the pentose (data not shown).

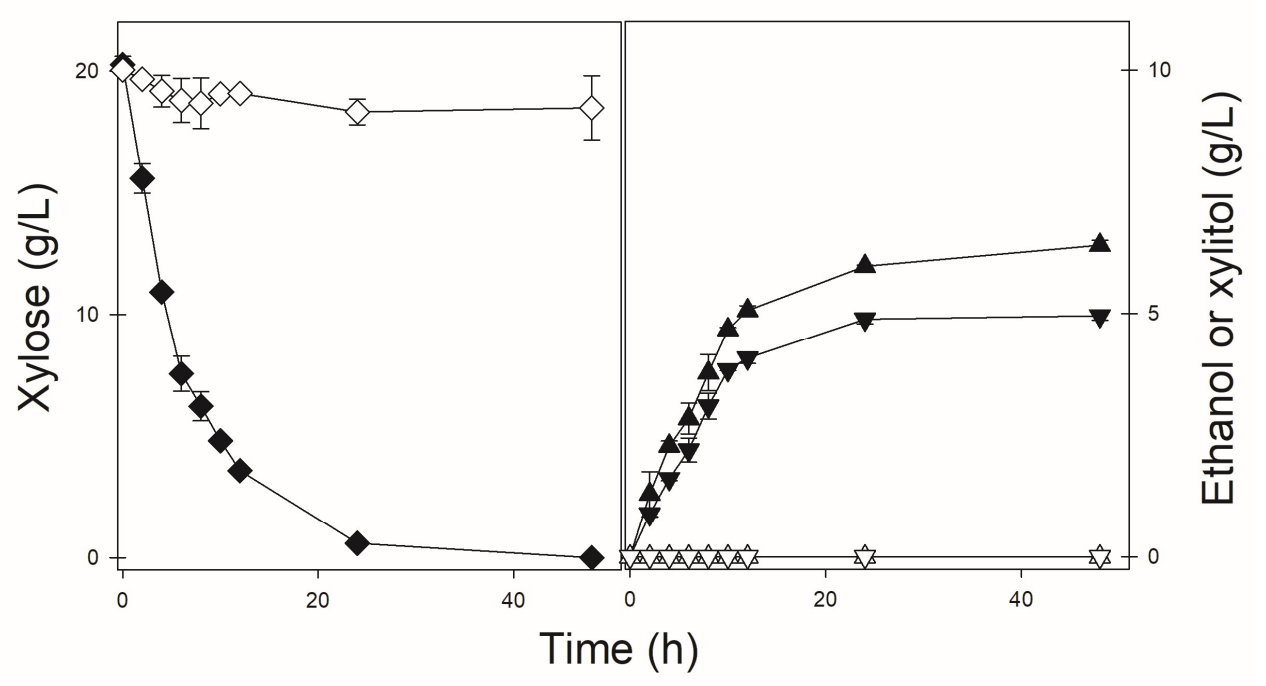

Figure 4. Xylose fermentation by the DLG-K1 strain transformed with the pPGK-SpXUT1 (open symbols) or pPGK-SpXUT1 $\Delta \mathrm{C}$ (black symbols) plasmids. The batch fermentation was performed with high cell concentrations (10 g DCW/L) and the amount of xylose (diamonds), ethanol (tringles) and xylitol (inverted triangles) in the medium were determined at the indicated time points as described in Materials and Methods

Since our results suggested that the ubiquitinylation of heterologous sugar permeases impair their successful expression in S. cerevisiae, and since this post-translational modification that triggers the endocytosis of the plasma membrane transporters depends on the activity of $\alpha$-arrestins that function as adaptors for the E3 ubiquitin ligase encoded by the essential gene RSP5 [50,51], we tested if deleting $\alpha$-arrestins involved in sugar transporter endocytosis could be another strategy to improve the functional expression of the cloned transporters in S. cerevisiae. Although this yeast has 14 known $\alpha$-arrestins, we 
focused in two $\alpha$-arrestins known to be involved in sugar transporter endocytosis: ROD1 (also known as ART4 for arrestin-related trafficking adaptors) which has been shown to mediate the ubiquitinylation and endocytosis of the high-affinity HXT6 and HXT4 glucose transporters [52-54], the low-affinity HXT1 and HXT3 glucose permeases [55], as well as the GAL2 galactose permease [56]; and its paralog ROG3 (also known as ART7) that has been shown to be implicated in the ubiquitinylation and endocytosis of the low-affinity HXT1 and HXT3 glucose permeases [54,55].

To verify the influence of these two $\alpha$-arrestins on the functionality of the SpXUT1 and SaXUT1 permeases in S. cerevisiae we constructed in our hxt-null DLG-K1 genetic background (Table 1) strains deleted in ROD1 (DLG-K1 $\triangle$ R1), ROG3 (DLG-K1 $\triangle$ R3), or both genes (DLG-K1 $\triangle R 1 \triangle R 3$ ). Figure 5 shows the growth patterns of these 3 strains, transformed with plasmids pGPD-SaXUT1 or pPGK-SpXUT1, in three carbon sources: maltose, glucose and xylose. Analyzing the data it is evident that the deletion of both $\alpha$-arrestins $(\operatorname{rod} 1 \Delta, \operatorname{rog} 3 \Delta)$ has a negative impact on growth even with the control carbon source ( $2 \%$ maltose). While all strains produced $8.7 \pm 0.7 \mathrm{~g}$ ethanol/L at the end of growth on this carbon source, strain DLG-K1 $\triangle \mathrm{R} 1 \Delta \mathrm{R} 3$ transformed with both plasmids produced less ethanol $(7.2 \pm 1.1 \mathrm{~g} / \mathrm{L})$ from $2 \%$ maltose. Deletion of both genes had also a negative impact when the cells were grown on the two other carbon sources.
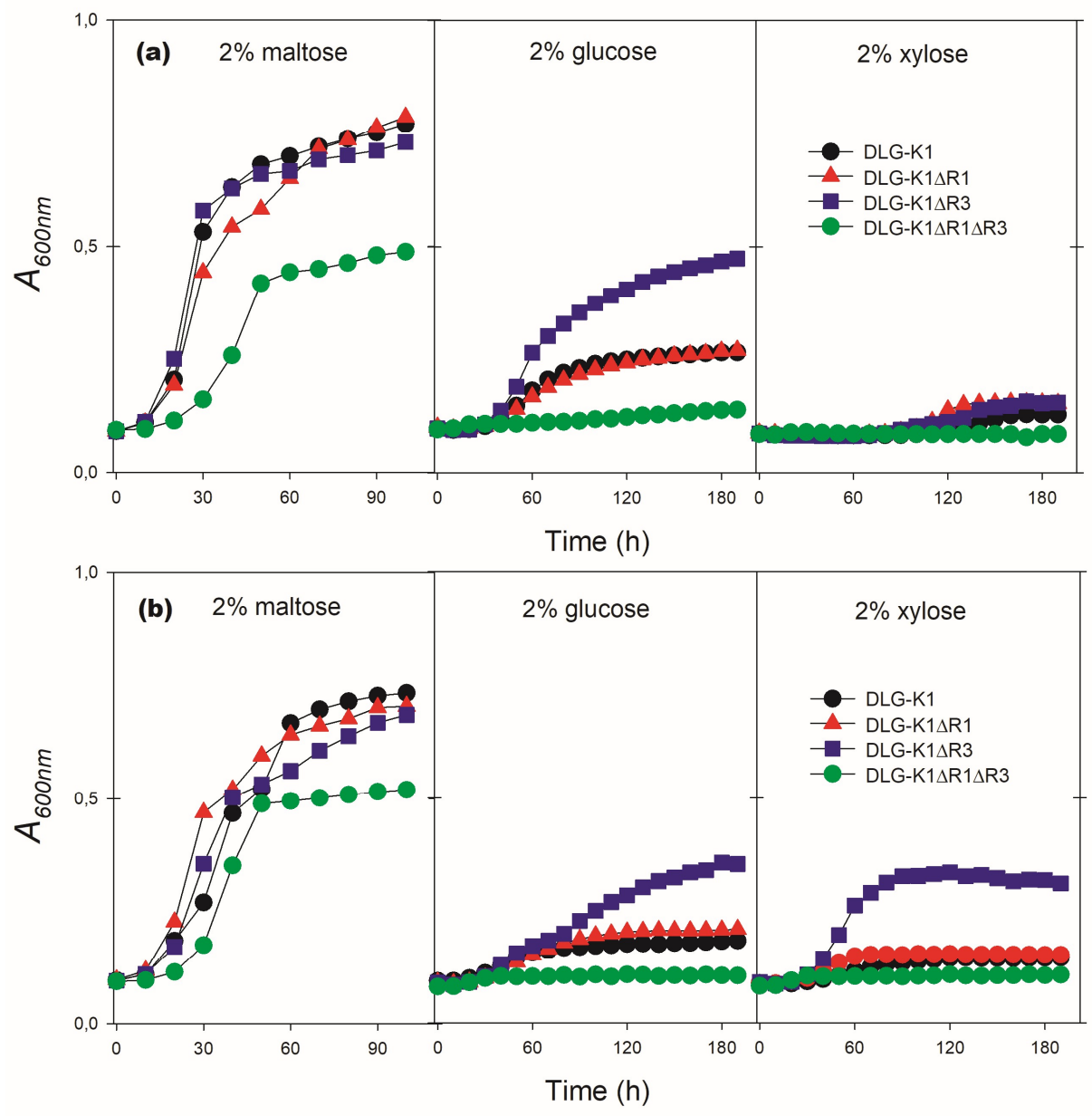

Figure 5. Growth of the indicated $h x t$-null strains containing the pPGK-SpXUT1 (a) or pGPD-SaXUT1 (b) plasmids on o-YNB medium containing $2 \%$ of the indicated carbon sources in a microplate reader as described in Materials and Methods. 
Growth on glucose was clearly improved in the rog $3 \Delta$ strain DLG-K1 $\Delta \mathrm{R} 3$ expressing both XUT1 permeases (Figure 5). The rog3 $\Delta$ cells expressing the SpXUT1 transporter produced $7.1 \pm 0.2 \mathrm{~g}$ ethanol/L at the end of growth on $2 \%$ glucose, while these same cells expressing the SaXUT1 permease produced only $1.1 \pm 0.1 \mathrm{~g}$ ethanol/L, a lower performance as already mentioned for cells expressing this permease. Regarding growth on xylose, for the cells expressing the SpXUT1 permease none of the strains deleted in ROD1 and/or ROG3 improved the utilization of this carbon source by the hxt-null strains, indicating that probably other $\alpha$-arrestins might be involved in ubiquitinylation of this transporter in the presence of the pentose. A different pattern was obtained with the strains expressing the SaXUT1 permease, as the DLG-K1 $\triangle$ R3 strain showed some growth on xylose (Figure 5), but without ethanol production. The improvements observed with the strains deleted in the two $\alpha$-arrestins shown in Figure 5 (performed in a microplate reader with 96-well plates) were confirmed when these strains DLG-K1 $\Delta$ R3 expressing both transporters were grown aerobically in cotton plugged Erlenmeyer flasks (data not shown).

\section{Discussion}

Our group had previously used the $h x t$-null strain DLG-K1 to clone and characterize three sugar transporters from Sc. stipitis that allowed growth and xylose fermentation by S. cerevisiae [27]. One of the transporters (SsXUT1) was already known to allow growth of this yeast on xylose [24], but the two others (SSHXT2.6 and SsQUP2) were completely new and even annotated for other functions in the published genome sequence of $S c$. stipitis [27]. An interesting characteristic of these three permeases is that, as shown here for the SsXUT1 permease (see Figure 3), they had only one lysine residue with high-confidence ubiquitinylation potential at their C-terminal cytoplasmic domain (SsXUT1 and SsQUP2), or at its N-terminal domain (SsHXT2.6). In contrast, the two putative sugar transporters cloned in this work from the xylose-fermenting yeasts $S p$. passalidarum and Sp. arborariae, having high sequence identity with SsXUT1, had several lysine residues with high-confidence ubiquitinylation potential at their C-terminal domain (e.g. SpXUT1), as well as lysine residues with medium-confidence ubiquitinylation potential also at their N-terminal cytoplasmic domain (see Figure 3).

In $S$. cerevisiae sugar transporters can be ubiquitinylated and removed from the plasma membrane through endocytosis depending not only on the type (and quantity) of the sugar present in the medium, but also in response to other environmental perturbations [49-51]. For example, low affinity and high capacity glucose transporters (e.g. HXT1 and HXT3) are endocytated in the absence of glucose (or presence of other carbon sources, like galactose, ethanol or lactate), while high affinity glucose transporters (e.g. HXT2, HXT6 and HXT7) are removed from the plasma membrane in the presence of excess glucose, but also in the absence of glucose (or presence of other carbon sources) [53,55,57-59]. The influence of ubiquitinylation and endocytosis in the expression of heterologous sugar transporters in S. cerevisiae is only starting to be understood [31].

Indeed, several efforts to express heterologous sugar transporters in this yeast have been hampered by the low success of such approaches. For example, Young and coworkers [24] cloned 23 transporters from different organisms (bacteria, plant and several yeasts) and expressed them in a $h x t$-null strain with a xylose utilization pathway. Of these 23 heterologous transporters, only 7 (including SsXUT1) conferred significant growth phenotypes on one or more of six different carbon sources tested. Several other recent manuscripts reporting the identification and cloning of sugar transporters from yeasts and fungi, with rates of successful expression of less than $1 / 3$ of the putative permeases in a $h x t$-null strain, are common [60-62]. Although there are many possible reasons for failure to efficiently express a putative sugar transporter in S. cerevisiae, including misfolding of the protein, problems with correct traffic of the permease to the plasma membrane, or even the lipid environment of the host, the stability at the cell surface is 
also of concern, and transporter ubiquitinylation and endocytosis is certainly an issue that could influence de heterologous expression of sugar transporters in this yeast.

Ubiquitinylation of lysine residues at the N- or C-terminal cytoplasmic domains are part of the molecular signals used for endocytic removal of sugar permeases, and several studies have shown that by mutating or deleting such residues the transporters are stabilized at the plasma membrane. For example, the S. cerevisiae HXT1 transporter has 4 lysine residues at its N-terminal domain involved in its ubiquitinylation and endocytosis, and truncating the whole N-terminal domain (deleting 56 residues to remove all 4 lysine residues) gives a functional transporter that is stable at the plasma membrane [58]. We have used recently this strategy to enhance xylose consumption and fermentation by an industrial S. cerevisiae strain [63], while site-directed mutation of these 4 residues in the HXT1 transporter was also shown to improve cell growth on this carbon source [64]. Another example involves one of the few yeast active xylose transporters characterized in S. cerevisiae, the Candida intermedia CiGXS1 gene encoding for a glucose/xylose $\mathrm{H}^{+}$-symporter [65]. This transporter in a hxt-null strain was submitted to several rounds of mutagenesis aiming to remove glucose inhibition, especially during glucose-xylose co-fermentations, and among several interesting mutations found there were some that truncated the C-terminal domain of the permease [66]. A systematic analysis of this C-terminal domain revealed that the best results in terms of growth on glucose plus xylose medium was when 27 amino acids were deleted from the end of the CiGXS1 permease, which included two lysine residues [66]. Finally, C-terminal truncations that enhance the stabilization and transport activity of two heterologous cellobiose transporters expressed in S. cerevisiae have been also reported, eliminating 1-2 lysine residues present at their C-terminal domains probably involved in ubiquitinylation and endocytosis [41]. Thus, our approach of truncating the SpXUT1 or SaXUT1 permeases, removing the potentially ubiquitinylation lysine residues at their $\mathrm{N}$ - and/or C-terminal domains, is certainly an interesting strategy to increase the repertory of heterologous sugar (especially pentose) transporters for molecular engineering approaches to improve biomass sugar utilization in recombinant $S$. cerevisiae strains for biofuel and chemical production.

Besides eliminating lysine residues from the cytoplasmic domains of the transporters by truncating their cytoplasmic terminal domains, we also tested the involvement of two $\alpha$-arrestins (ROD1 and ROG3) known to be involved in sugar transporters ubiquitinylation and endocytosis in S. cerevisiae. While deletion of ROD1 or both $\alpha$-arrestins in our hxt-null strain had no effect on the absence of growth of the strains expressing the two XUT1 permeases, deletion of ROG3 had a positive effect with both permeases during growth on glucose, but during growth on xylose only the SaXUT1-expressing strain DLG-K1 $\Delta$ R3 had its growth enhanced (Figure 5). This more complex situation might reflect the fact that most $\alpha$-arrestins have overlapping functions when promoting the ubiquitinylation of the permeases by the RSP5 ubiquitin ligase, and many transporters are regulated by two or even up to four $\alpha$-arrestins, reflecting the diverse signaling pathways that mediate transporter ubiquitinylation and endocytosis triggered by changing environmental conditions [41,50,51,54-56]. Nevertheless, our results highlight the involvement of the ROG3 $\alpha$-arrestin in down-regulation of the heterologous SpXUT1 and SaXUT1 permeases expressed in S. cerevisiae in the presence of glucose, while a more complex situation is evident when xylose is the carbon source for growth.

\section{Conclusions}

In the present work we have cloned two XUT1 permeases from the xylose-fermenting yeasts $S p$. passalidarum and $S p$. arborariae. While these two transporters seemed to be not functional in S. cerevisiae, the truncation of the $\mathrm{N}$ - and/or C-terminal domains, eliminating lysine residues with ubiquitinylation potential, allowed the functional expression of the transporters in an hxt-null strain. Thus, this might be an interesting strategy to increase the repertory of heterologous sugar transporters for molecular engineering approaches to improve biomass sugar utilization by $S$. cerevisiae. This work 
therefore highlights the importance of post-translational modifications in the correct expression of novel sugar transporters in recombinant $S$. cerevisiae strains, aiming at an efficient $2 \mathrm{G}$ bioethanol production.

Author Contributions: Conceptualization, M.M.K., A.A.S. and B.U.S.; investigation, methodology, and formal analysis, M.M.K., A.A.S., L.G.K., F.G., and M.J.L.; resources, C.F., M.J.L. and B.U.S.; writing-review and editing, M.J.L. and B.U.S.; project administration and funding acquisition, C.F. and B.U.S. All authors have read and agreed to the published version of the manuscript.

Funding: This work was supported in part by grants and fellowships from the Brazilian agencies CAPES/FCT (process no 359/14), CNPq (process no 490029/2009-4, 551392/2010-0, 307290/2012-3, 478841/2013-2, 30862/2015-6 and 308389/2019-0), and FINEP (process n ${ }^{\circ}$ 01.09.0566.00/1421-08), and also by the Fundação para a Ciência e a Tecnologia (FCT), Portugal (postdoctoral fellowship SFRH/BPD/102803/2014 to M.J.L.) and I.P., Project MOSTMICRO-ITQB..

Acknowledgments: We would like to thank Dr. Carlos A. Rosa (Federal University of Minas Gerais, Brazil) for providing the Spathaspora yeast strains used in the present work.

Conflicts of Interest: The authors declare no conflict of interest. The funders had no role in the design of the study; in the collection, analyses, or interpretation of data; in the writing of the manuscript, or in the decision to publish the results.

\section{References}

1. Goldemberg, J. Ethanol for a sustainable energy future. Science 2007, 315, 808-810. doi: 10.1126/science.1137013

2. Jaiswal, D.; de Souza, A.; Larsen, S.; Lebauer, D.S.; Miguez, F.E.; Sparovek, G.; Bollero, G.; Buckeridge, M.S.; Long, S.P. Brazilian sugarcane ethanol as an expandable green alternative to crude oil use. Nature Clim. Change 2017, 7, 788-792. doi: 10.1038/nclimate3410

3. Liu, Y.; Cruz-Morales, P.; Zargar, A.; Belcher, M.S.; Pang, B.; Englund, E.; Dan, Q.; Yin, K.; Keasling, J.D. Biofuels for a sustainable future. Cell 2021, 184, 1636-1647. doi: 10.1016/j.cell.2021.01.052

4. Jacobus, A.P.; Gross, J.; Evans, J.H.; Ceccato-Antonini, S.R.; Gombert, A.K. Saccharomyces cerevisiae strains used industrially for bioethanol production. Essays Biochem. 2021, 65, 147-161. doi: 10.1042/EBC20200160

5. Trichez, D.; Bergmann, J.C.; Calsing, L.C.G.; Cançado, L.J. How many bioethanol generations can we have?. In Ethanol as a Green Alternative Fuel: Insight and Perspectives, $1^{\text {st }}$ ed.; Treichel, H., Alves, S.L., Jr., Fongaro, G., Müller, C., Eds.; Nova Science Publishers Inc.: Hauppauge, NY, USA, 2019; pp. 21-55

6. dos Santos, L.V.; de Barros Grassi, M.C.; Gallardo, J.C.M.; Pirolla, R.A.S.; Calderón, L.L.; de Carvalho-Netto, O.V.; Parreiras, L.S.; Camargo, E.L.O.; Drezza, A.L.; Missawa, S.K.; Teixeira, G.S.; Lunardi, I.; Bressiani, J.; Pereira, G.A.G. Second-generation ethanol: the need is becoming a reality. Ind. Biotechnol. 2016, 12, 40-57. doi: 10.1089/ind.2015.0017

7. Gao, M.; Ploessl, D.; Shao, Z. Enhancing the co-utilization of biomass-derived mixed sugars by yeasts. Front. Microbiol. 2019, 9 , 3264. doi: 10.3389/fmicb.2018.03264

8. Kim, J.; Hwang, S.; Lee, S.M. Metabolic engineering for the utilization of carbohydrate portions of lignocellulosic biomass. Metab. Eng. 2021, in-press. doi: 10.1016/j.ymben.2021.10.002

9. Gírio, F.M.; Fonseca, C.; Carvalheiro, F.; Duarte, L.C.; Marques, S.; Bogel-Łukasik, R. Hemicelluloses for fuel ethanol: A review. Bioresour. Technol. 2010, 101, 4775-4800. doi: 10.1016/j.biortech.2010.01.088

10. da Silva, A.S.; Espinheira, R.P.; Teixeira, R.S.S.; de Souza, M.F.; Ferreira-Leitão, V.; Bon, E.P.S. Constraints and advances in high-solids enzymatic hydrolysis of lignocellulosic biomass: a critical review. Biotechnol. Biofuels. 2020, 13, 58. doi: 10.1186/s13068-020-01697-w

11. Stambuk, B.U. Yeasts: The leading figures on bioethanol production. In Ethanol as a Green Alternative Fuel: Insight and Perspectives, $1^{\text {st }}$ ed.; Treichel, H.; Alves-Jr, S.L.; Fongaro, G.; Müller, C.; Eds.; Nova Science Publishers Inc.: Hauppauge, NY, USA, 2019, pp 57-91

12. Patiño, M.A.; Ortiz, J.P.; Velásquez, M.; Stambuk, B.U. D-Xylose consumption by nonrecombinant Saccharomyces cerevisiae: A review. Yeast 2019, 36, 541-556. doi: 10.1002/yea.3429

13. Ye, S.; Kim, J.W.; Kim, S.R. Metabolic engineering for improved fermentation of L-arabinose. J. Microbiol. Biotechnol. 2019, 29, 339-346. doi: 10.4014/jmb.1812.12015

14. Sharma, S.; Arora, A. Tracking strategic developments for conferring xylose utilization/fermentation by Saccharomyces cerevisiae. Ann. Microbiol. 2020, 70, 50. doi: 10.1186/s13213-020-01590-9

15. Jin, Y.S.; Lee, T.H.; Choi, Y.D.; Ryu, Y.W.; Seo, J.H. Conversion of xylose to ethanol by recombinant Saccharomyces cerevisiae containing genes for xylose reductase and xylitol dehydrogenase from Pichia stipitis. J. Microb. Biotechnol. 2000, 10, 564-567

16. Cadete, R.M.; De Las Heras, A.M.; Sandström, A.G.; Ferreira, C.; Gírio, F.; Gorwa-Grauslund, M.F.; Rosa, C.A.; Fonseca, C.. Exploring xylose metabolism in Spathaspora species: XYL1.2 from Spathaspora passalidarum as the key for efficient anaerobic 
xylose fermentation in metabolic engineered Saccharomyces cerevisiae. Biotechnol. Biofuels 2016, 9, 167. doi: 10.1186/s13068-016-0570-6

17. Mouro, A.; dos Santos, A.A.; Agnolo, D.D.; Gubert, G.F.; Bon, E.P.S.; Rosa, C.A.; Fonseca, C.; Stambuk, B.U. Combining xylose reductase from Spathaspora arborariae with xylitol dehydrogenase from Spathaspora passalidarum to promote xylose consumption and fermentation into xylitol by Saccharomyces cerevisiae. Fermentation 2020, 6, 72. doi: 10.3390/fermentation6030072

18. Brat, D.; Boles, E.; Wiedemann, B. Functional expression of a bacterial xylose isomerase in Saccharomyces cerevisiae. Appl. Environ. Microbiol. 2009, 75, 2304-2311. doi: 10.1128/AEM.02522-08

19. Cunha, J.T.; Soares, P.O.; Romaní, A.; Thevelein, J.M.; Domingues, L. Xylose fermentation efficiency of industrial Saccharomyces cerevisiae yeast with separate or combined xylose reductase/xylitol dehydrogenase and xylose isomerase pathways. Biotechnol. Biofuels 2019, 12, 20. doi: 10.1186/s13068-019-1360-8

20. Jeong, D.; Oh, E.J.; Ko, J.K.; Nam, J.-O.; Park, H.S.; Jin, Y.-S.; Lee, E.J.; Kim, S.R. Metabolic engineering considerations for the heterologous expression of xylose-catabolic pathways in Saccharomyces cerevisiae. PLoS ONE 2020, 15: e0236294. doi: 10.1371/journal.pone.0236294

21. Gonçalves, D.L.; Matsushika, A.; de Sales, B.B.; Goshima, T.; Bon, E.P.S.; Stambuk, B.U. Xylose and xylose/glucose co-fermentation by recombinant Saccharomyces cerevisiae strains expressing individual hexose transporters. Enzyme Microb. Technol. 2014, 63, 13-20. doi: 10.1016/j.enzmictec.2014.05.003

22. Knoshaug, E.P.; Franden, M.A.; Stambuk, B.U.; Zhang, M.; Singh, A. Utilization and transport of L-arabinose by non-Saccharomyces yeasts. Cellulose 2009, 16, 729-741. doi: 10.1007/s10570-009-9319-8

23. Fonseca, C.; Olofsson, K.; Ferreira, C.; Runquist, D.; Fonseca, L.L.; Hahn-Hägerdal, B.; Lidén, G.. The glucose/xylose facilitator Gxf1 from Candida intermedia expressed in a xylose-fermenting industrial strain of Saccharomyces cerevisiae increases xylose uptake in SSCF of wheat straw. Enzyme Microb. Technol. 2011, 48, 518-525. doi: 10.1016/j.enzmictec.2011.02.010

24. Young, E.; Poucher, A.; Comer, A.; Bailey, A.; Alper, H. Functional survey for heterologous sugar transport proteins, using Saccharomyces cerevisiae as a host. Appl. Environ. Microbiol. 2011, 77, 3311-3319. doi: 10.1128/AEM.02651-10

25. Moon, J.; Liu, Z.L.; Ma, M.; Slininger, P.J. New genotypes of industrial yeast Saccharomyces cerevisiae engineered with YXI and heterologous xilose transporters improve xylose utilization and ethanol production. Biocatal. Agricult. Biotechnol. 2013, 2, 247-254. doi: 10.1016/j.bcab.2013.03.005

26. Knoshaug, E.P.; Vidgren, V.; Magalhães, F.; Jarvis, E.E.; Franden, M.A.; Zhang, M.; Singh, A. Novel transporters from Kluyveromyces marxianus and Pichia guilliermondii expressed in Saccharomyces cerevisiae enable growth on L-arabinose and D-xylose. Yeast 2015, 32, 615-628. doi: 10.1002/yea.3084

27. de Sales, B.B.; Scheid, B.; Gonçalves, D.L.; Knychala, M.M.; Matsushika, A.; Bon, E.P.; Stambuk, B.U. Cloning novel sugar transporters from Scheffersomyces (Pichia) stipitis allowing D-xylose fermentation by recombinant Saccharomyces cerevisiae. Biotechnol. Lett. 2015, 37, 1973-1982. doi: 10.1007/s10529-015-1893-2

28. Jojima, T.; Omumasaba, C.A.; Inui, M.; Yukawa, H. Sugar transporters in efficient utilization of mixed sugar substrates: current knowledge and outlook. Appl, Microbiol, Biotechnol. 2010, 85, 471-480. doi: 10.1007/s00253-009-2292-1

29. Hara, K.Y.; Kobayashi, J.; Yamada, R.; Sasaki, D.; Kuriya, Y.; Hirono-Hara, Y.; Ishii, J.; Araki, M.; Kondo, A. Transporter engineering in biomass utilization by yeast. FEMS Yeast Res. 2017, 17, fox061. doi: 10.1093/femsyr/fox061

30. Sharma, N.K.; Behera, S.; Arora, R.; Kumar, S.; Sani, R.K. Xylose transport in yeast for lignocellulosic ethanol production: Current status. J. Biosci. Bioeng. 2018, 125, 259-267. doi: 10.1016/j.jbiosc.2017.10.006

31. Nijland, J.G.; Driessen, A.J.M. Engineering of pentose transport in Saccharomyces cerevisiae for biotechnological applications. Front. Bioeng. Biotechnol. 2020, 7, 464. doi: 10.3389/fbioe.2019.00464

32. Wieczorke, R.; Krampe, S.; Weierstall, T.; Freidel, K.; Hollenberg, C.P.; Boles, E. Concurrent knock-out of at least 20 transporter genes is required to block uptake of hexoses in Saccharomyces cerevisiae. FEBS Lett. 1999, 464, 123-128. doi: 10.1016/s0014-5793(99)01698-1

33. Boles, E.; Oreb, M. A growth-based screening system for hexose transporters in yeast. Methods Mol. Biol. 2018, 1713, $123-135$. doi: 10.1007/978-1-4939-7507-5_10

34. Hamacher, T.; Becker, J.; Gárdonyi, M.; Hahn-Hägerdal, B.; Boles, E. Characterization of the xylose-transporting properties of yeast hexose transporters and their influence on xylose utilization. Microbiology 2002; 148, 2783-2788. doi: 10.1099/00221287-148-9-2783

35. Solis-Escalante, D.; van den Broek, M.; Kuijpers, N.G.; Pronk, J.T.; Boles, E.; Daran, J.M.; Daran-Lapujade, P. The genome sequence of the popular hexose-transport-deficient Saccharomyces cerevisiae strain EBY.VW4000 reveals LoxP/Cre-induced translocations and gene loss. FEMS Yeast Res. 2015, 15, fou004. doi: 10.1093/femsyr/fou004

36. Wijsman, M.; Swiat, M.A.; Marques, W.L.; Hettinga, J.K.; van den Broek, M.; Torre Cortés, P.; Mans, R.; Pronk, J.T.; Daran, J.M.; Daran-Lapujade, P. A toolkit for rapid CRISPR-SpCas9 assisted construction of hexose-transport-deficient Saccharomyces cerevisiae strains. FEMS Yeast Res. 2019, 19, foy107. doi: 10.1093/femsyr/foy107

37. Nguyen, N.H.; Suh, S.O.; Marshall, C.J.; Blackwell, M. Morphological and ecological similarities: wood-boring beetles associated with novel xylose-fermenting yeasts, Spathaspora passalidarum gen. sp. nov. and Candida jeffriesii sp. nov. Mycol. Res. 2006, 110, 1232-1241. doi: 10.1016/j.mycres.2006.07.002

38. Wohlbach, D.J.; Kuo, A.; Sato, T.K.; Potts, K.M.; Salamov, A.A.; Labutti, K.M.; Sun, H.; Clum, A.; Pangilinan, J.L.; Lindquist, E.A.; Lucas, S.; Lapidus, A.; Jin, M.; Gunawan, C.; Balan, V.; Dale, B.E.; Jeffries, T.W.; Zinkel, R.; Barry, K.W.; Grigoriev, I.V.; 
Gasch, A.P. Comparative genomics of xylose-fermenting fungi for enhanced biofuel production. Proc. Natl. Acad. Sci. USA 2011, 108, 13212-13217. doi: 10.1073/pnas.1103039108

39. Cadete, R.M.; Santos, R.O.; Melo, M.A.; Mouro, A.; Gonçalves, D.L.; Stambuk, B.U.; Rosa, C.A. Spathaspora arborariae sp. nov., a D-xylose fermenting yeast species isolated from rotting wood in Brazil. FEMS Yeast Res. 2009, 9, 1338-1342. doi: 10.1111/j.1567-1364.2009.00582.x

40. Lobo, F.P.; Gonçalves, D.L.; Alves-Jr, S.L.; Gerber, A.L.; Vasconcelos, A.T.R.; Basso, L.C.; Franco, G.R., Soares, M.A.; Cadete, R.M.; Rosa, C.A.; Stambuk, B.U. Draft genome sequence of the D-xylose-fermenting yeast Spathaspora arborariae UFMG-HM19.1A ${ }^{\mathrm{T}}$. Genome Announc. 2014, 2, e01163-13. doi: 10.1128/genomeA.01163-13

41. Sen, A.; Acosta-Sampson, L.; Alvaro, C.G.; Ahn, J.S.; Cate, J.H.; Thorner, J. Internalization of heterologous sugar transporters by endogenous $\alpha$-arrestins in the yeast Saccharomyces cerevisiae. Appl. Environ. Microbiol. 2016, 82, 7074-7085. doi: 10.1128/AEM.02148-16

42. Petracek, M.E.; Longtine, M.S. PCR-based engineering of yeast genome. Methods Enzymol. 2002, 350, 445-469.

43. Kang YS, Kane J, Kurjan K, Stadel JM, Tipper DJ. Effects of expression of mammalian G alpha and hybrid mammalian-yeast G alpha proteins on the yeast pheromone response signal transduction pathway. Mol. Cell. Biol. 1990, 10, $2582-2590$.

44. Mumberg, D.; Müller, R.; Funk, M. Yeast vectors for the controlled expression of heterologous proteins in different genetic backgrounds. Gene 1995, 156, 119-122.

45. Ausubel, F.M.; Brent, R.; Kingston, R.E.; Moore, D.D.; Seidman, J.G.; Smith, J.A.; Struhl, K. Short Protocols in Molecular Biology, $3^{\text {rd }}$ ed.; John Wiley \& Sons: New York, USA, 1995.

46. Gietz, D.; St Jean, A.; Woods, R.A.; Schiestl, R.H. Improved method for high efficiency transformation of intact yeast cells. Nucleic Acids Res. 1992, 20, 1425.

47. Sievers, F.; Higgins, D.G. Clustal Omega for making accurate alignments of many protein sequences. Protein Sci. 2018, 27, 135-145. doi: 10.1002/pro.3290.

48. Radivojac, P.; Vacic, V.; Haynes, C.; Cocklin, R.R.; Mohan, A.; Heyen, J.W.; Goebl, M.G.; Iakoucheva, L.M. Identification, analysis, and prediction of protein ubiquitination sites. Proteins 2010, 78, 365-380. doi: 10.1002/prot.22555.

49. Horák, J. The role of ubiquitin in down-regulation and intracellular sorting of membrane proteins: insights from yeast. Biochim. Biophys. Acta. 2003, 1614, 139-155. doi: 10.1016/s0005-2736(03)00195-0.

50. Kahlhofer, J.; Leon, S.; Teis, D.; Schmidt, O. The $\alpha$-arrestin family of ubiquitin ligase adaptors links metabolism with selective endocytosis. Biol. Cell. 2021, 113, 183-219. doi: 10.1111/boc.202000137.

51. Barata-Antunes, C.; Alves, R.; Talaia, G.; Casal, M.; Gerós, H.; Mans, R.; Paiva, S. Endocytosis of nutrient transporters in fungi: The ART of connecting signaling and trafficking. Comput. Struct. Biotechnol. J. 2021, 19, 1713-1737. doi: 10.1016/j.csbj.2021.03.013.

52. Nikko, E.; Pelham, H.R. Arrestin-mediated endocytosis of yeast plasma membrane transporters. Traffic 2009, 10, 1856-1867. doi: 10.1111/j.1600-0854.2009.00990.x.

53. Llopis-Torregrosa, V.; Ferri-Blázquez, A.; Adam-Artigues, A.; Deffontaines, E.; van Heusden, G.P.; Yenush, L. Regulation of the yeast Hxt6 hexose transporter by the Rod1 $\alpha$-arrestin, the Snf1 protein kinase, and the Bmh2 14-3-3 protein. J. Biol. Chem. 2016, 291, 14973-14985. doi: 10.1074/jbc.M116.733923.

54. Savocco, J.; Nootens, S.; Afokpa, W.; Bausart, M.; Chen, X.; Villers, J.; Renard, H.F.; Prévost, M.; Wattiez, R.; Morsomme, P. Yeast $\alpha$-arrestin Art2 is the key regulator of ubiquitylation-dependent endocytosis of plasma membrane vitamin B1 transporters. PLoS Biol. 2019, 17, e3000512. doi: 10.1371/journal.pbio.3000512.

55. O'Donnell, A.F.; McCartney, R.R.; Chandrashekarappa, D.G.; Zhang, B.B.; Thorner, J.; Schmidt, M.C. 2-Deoxyglucose impairs Saccharomyces cerevisiae growth by stimulating Snf1-regulated and $\alpha$-arrestin-mediated trafficking of hexose transporters 1 and 3. Mol. Cell. Biol. 2015, 35, 939-955. doi: 10.1128/MCB.01183-14.

56. Tamayo Rojas, S.A.; Schmidl, S.; Boles, E.; Oreb, M. Glucose-induced internalization of the S. cerevisiae galactose permease Gal2 is dependent on phosphorylation and ubiquitination of its aminoterminal cytoplasmic tail. FEMS Yeast Res. 2021, 21, foab019. doi: 10.1093/femsyr/foab019.

57. Kruckeberg, A.L.; Ye, L.; Berden, J.A.; van Dam, K. Functional expression, quantification and cellular localization of the Hxt2 hexose transporter of Saccharomyces cerevisiae tagged with the green fluorescent protein. Biochem. J. 1999, 339, $299-307$.

58. Roy, A.; Kim, Y.B.; Cho, K.H.; Kim, J.H. Glucose starvation-induced turnover of the yeast glucose transporter Hxt1. Biochim. Biophys. Acta 2014, 1840, 2878-2885. doi: 10.1016/j.bbagen.2014.05.004.

59. Hovsepian, J.; Defenouillère, Q.; Albanèse, V.; Váchová, L.; Garcia, C.; Palková, Z.; Léon, S. Multilevel regulation of an $\alpha$-arrestin by glucose depletion controls hexose transporter endocytosis. J. Cell. Biol. 2017, 216, 1811-1831. doi: 10.1083/jcb.201610094.

60. Lazar, Z.; Neuvéglise, C.; Rossignol, T.; Devillers, H.; Morin, N.; Robak, M.; Nicaud, J.M.; Crutz-Le Coq, A.M. Characterization of hexose transporters in Yarrowia lipolytica reveals new groups of Sugar Porters involved in yeast growth. Fungal Genet. Biol. 2017, 100, 1-12. doi: 10.1016/j.fgb.2017.01.001.

61. Liang, Z.; Liu, D.; Lu, X.; Zong, H.; Song, J.; Zhuge, B. Identification and characterization from Candida glycerinogenes of hexose transporters having high efficiency at high glucose concentrations. Appl. Microbiol. Biotechnol. 2018, 102, 5557-5567. doi: 10.1007/s00253-018-9027-0.

62. Jiang, Y.; Shen, Y.; Gu, L.; Wang, Z.; Su, N.; Niu, K.; Guo, W.; Hou, S.; Bao, X.; Tian, C.; Fang, X. Identification and characterization of an efficient D-xylose transporter in Saccharomyces cerevisiae. J. Agric. Food Chem. 2020, 68, 2702-2710. doi: 10.1021/acs.jafc.9b07113. 
63. Pereira, I.O.; dos Santos, A.A.; Gonçalves, D.L.; Purificação, M.; Guimarães, N.C.; Tramontina, R.; Coutouné, N.; Zanella, E.; Matsushika, A.; Stambuk, B.U.; Ienczak, J.L. Comparison of Spathaspora passalidarum and recombinant Saccharomyces cerevisiae for integration of first- and second-generation ethanol production. FEMS Yeast Res. 2021, 21, foab048. doi: 10.1093/femsyr/foab048.

64. Nijland, J.G.; Vos, E.; Shin, H.Y.; de Waal, P.P.; Klaassen, P.; Driessen, A.J. Improving pentose fermentation by preventing ubiquitination of hexose transporters in Saccharomyces cerevisiae. Biotechnol. Biofuels. 2016, 9, 158. doi: 10.1186/s13068-016-0573-3.

65. Leandro, M.J.; Gonçalves, P.; Spencer-Martins, I. Two glucose/xylose transporter genes from the yeast Candida intermedia: first molecular characterization of a yeast xylose- $\mathrm{H}^{+}$symporter. Biochem. J. 2006, 395, 543-549. doi: 10.1042/BJ20051465.

66. Li, H.; Schmitz, O.; Alper, H.S. Enabling glucose/xylose co-transport in yeast through the directed evolution of a sugar transporter. Appl. Microbiol. Biotechnol. 2016, 100, 10215-10223. doi: 10.1007/s00253-016-7879-8. 